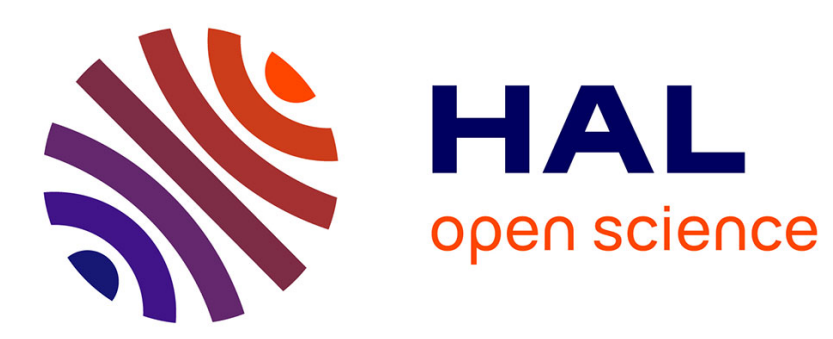

\title{
Physical model for static and dynamic scaling in spin glasses
}

\author{
A. P Malozemoff, B. Barbara
}

\section{To cite this version:}

A. P Malozemoff, B. Barbara. Physical model for static and dynamic scaling in spin glasses. Journal of Applied Physics, 1985, 57 (8), pp.3410 - 3412. 10.1063/1.335057 . hal-01660065

\section{HAL Id: hal-01660065 \\ https://hal.science/hal-01660065}

Submitted on 9 Dec 2017

HAL is a multi-disciplinary open access archive for the deposit and dissemination of scientific research documents, whether they are published or not. The documents may come from teaching and research institutions in France or abroad, or from public or private research centers.
L'archive ouverte pluridisciplinaire HAL, est destinée au dépôt et à la diffusion de documents scientifiques de niveau recherche, publiés ou non, émanant des établissements d'enseignement et de recherche français ou étrangers, des laboratoires publics ou privés. 


\title{
AIP Applied Physics
}

\section{Physical model for static and dynamic scaling in spin glasses}

\author{
A. P. Malozemoff and B. Barbara
}

Citation: J. Appl. Phys. 57, 3410 (1985); doi: 10.1063/1.335057

View online: http://dx.doi.org/10.1063/1.335057

View Table of Contents: http://jap.aip.org/resource/1/JAPIAU/v57/i8

Published by the American Institute of Physics.

\section{Related Articles}

The competing spin orders and fractional magnetization plateaus of the classical Heisenberg model on ShastrySutherland lattice: Consequence of long-range interactions

J. Appl. Phys. 113, 073908 (2013)

Microscopic theory of the glassy dynamics of passive and active network materials

J. Chem. Phys. 138, 12A521 (2013)

Ferroelectricity enhanced by disorder in collinear-magnetism-induced multiferroic state

J. Appl. Phys. 112, 114110 (2012)

Critical behavior of two-dimensional spin systems under the random-bond six-state clock model

J. Appl. Phys. 112, 063924 (2012)

The role of dipole-dipole interaction in modulating the step-like magnetization of Ca3Co2O6

J. Appl. Phys. 111, 07E133 (2012)

\section{Additional information on J. Appl. Phys.}

Journal Homepage: http://jap.aip.org/

Journal Information: http://jap.aip.org/about/about_the_journal

Top downloads: http://jap.aip.org/features/most_downloaded

Information for Authors: http://jap.aip.org/authors

\section{ADVERTISEMENT}

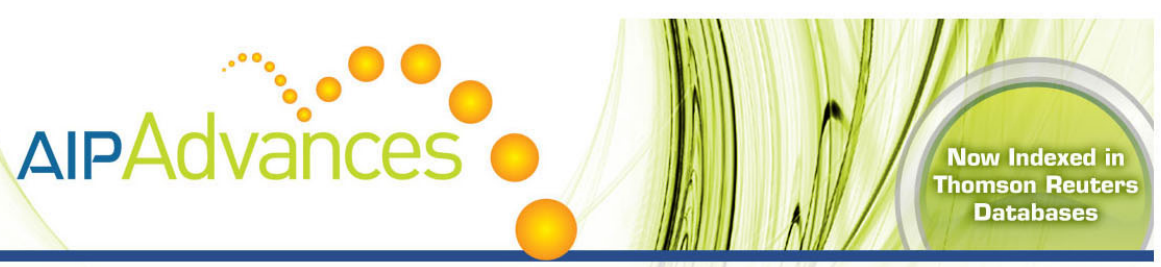

\section{Explore AIP's open access journal: •Rapid publication \\ - Article-level metrics \\ - Post-publication rating and commenting}




\title{
Physical model for static and dynamic scaling in spin glasses
}

\author{
A. P. Malozemoff \\ IBM T. J. Watson Research Center, Yorktown Heights, New York 10598
}

B. Barbara

CNRS Lab. Louis Néel, 166X-38042-Grenoble, France

\begin{abstract}
We extend earlier critical fractal cluster models of a spin glass phase transition. At the transition temperature in finite field, a negative excluded-volume term dominates a positive saturatedcluster term, giving the negative $H^{2 / \delta}$ nonlinear susceptibility observed experimentally. The two competing effects in general have different relaxation times and therefore novel implications for dynamic scaling.
\end{abstract}

In this paper we extend earlier critical fractal cluster models of spin glasses ${ }^{1-8}$ to explain the negative nonlinear equilibrium susceptibility observed at the phase transition temperature $e^{9,10}$ and to suggest an interpretation of dynamic scaling phenomena as well. ${ }^{11,12}$ The first critical cluster models by Smith ${ }^{1}$ and Abrikosov ${ }^{3}$ envisioned a phase transition caused by percolation of an infinite cluster, but the lack of frustration in their models led to the prediction of standard site percolation exponents which are far from experimentally observed values. ${ }^{13}$ Binder ${ }^{2}$ pointed out, however, that because of frustration the cluster size distribution is likely to be very different from that of the site percolation problem; this suggests the possibility of different exponents. ${ }^{5}$

Abrikosov's work remains particularly valuable because he introduced a quite general scaling form for the distribution of cluster sizes, closely related to the distribution proposed by Stauffer ${ }^{14}$ which we use here: The number $n_{s}$ of clusters (per magnetic site) containing $s$ spins is, in Stauffer's notation,

$$
n_{s}=s^{-\tau} f_{s}\left(s / s_{\xi}\right), \quad \tau=2+\delta^{-1} .
$$

Here $\delta$ is a standard critical exponent, $f_{s}$ is a distribution function which approaches a constant at low values of $s$, and $s_{\xi}$ is a characteristic cluster size related to the correlation length $\xi$ and the clusters' fractal dimension $D$ by $s_{\xi} \propto \xi^{D}$. A typical form for the distribution of spin numbers $s n_{s}$ is shown in Fig. 1, with $s_{\xi}$ marking the cutoff of $f_{s}$. Such a distribution applies in a critical region of reduced temperature $\epsilon=\left(T-T_{G}\right) / T_{G}$ and field $H$ around a phase transition at $T_{G}$ and at $H=0$. In the spirit of earlier work, the spins of classical moment $\mu_{0}$ are assumed rigidly coupled within the clusters, so that the cluster moment is $\mu_{0} s^{y}$ where $y=1 / 2$ for a spin glass cluster with perfectly random orientations and $y=1$ for a ferromagnet. Interactions between clusters ${ }^{15}$ are ignored. While there is no rigorous argument for these assumptions, they do lead to results in good agreement with scaling theory and with experiment, as we shall summarize below. A crude argument for ignoring the polarizability of clusters is that because of their ramified, fractal shape, they are porous, and so any polarizability can be associated with the interpenetrating paramagnetic soup. The crude argument for ignoring interactions is that relevant interactions are taken into account by the critical growth of the clusters themselves as the phase transition is approached.

Because the clusters are assumed rigid and noninteracting, they respond only to a field, and only by rotation or a change in cluster size. Then the susceptibility is given by the classical formula of superparamagnetism

$$
\chi=N H^{-1} \int d s n_{s} \mu_{0} s^{y} \mathscr{L}\left(\mu_{0} s^{y} H / k T\right),
$$

where $N$ is the number density of spins and $\mathscr{L}$ is the langevin function.

To normalize the distribution (1) in view of the divergence of $s^{1-\tau}$ as $s \rightarrow 0$, we follow Abrikosov ${ }^{3}$ by taking

$$
\begin{aligned}
& \int_{0}^{\infty} d s s n_{s} \sim \int_{0}^{s_{\xi}} d s s^{1-\tau}=1, \quad \epsilon \geqslant 0, \\
& \int_{0}^{s_{\xi}} d s s^{1-\tau}=1-\delta s_{\xi}^{2-\tau}, \quad \epsilon \leqslant 0, \quad H=0 .
\end{aligned}
$$

In effect, the lower limit of the integration is adjusted because single spins act as a reservoir for the growing clusters.

In zero field, we expect $\xi \propto|\epsilon|^{-\nu}$ and hence $s_{\xi} \propto|\epsilon|^{-\nu D}$, where $v$ is another standard critical exponent. The combined exponent $v D$ is just the crossover exponent $\phi(=1 / \sigma$ in Stauffer's notation i $")$ defined by the characteristic scaling variable $H^{1 / y} \xi^{-\cdots}$. [Ote way to see this is to compare the scaling variables ${ }^{y} H$ in the Langevin function of $\mathrm{Eq}$. (2) to $H^{1 / y} \epsilon^{-\phi}$ and to substitute $s \propto \epsilon^{-\nu D}$. T Thus, we see that as $\epsilon \rightarrow 0$ from above, $s_{\xi} \rightarrow \infty$ and the cluster distribution $s n_{s}$ approaches the simple power-law distribution $s^{1-\tau}$ (see dashed line in Fig. 1). As $\epsilon$ decreases below zero, $s_{\xi} \propto|\epsilon|^{-\phi}$ decreases while an infinite percolating cluster grows. It is natural to identify the spin fraction of the infinite cluster as

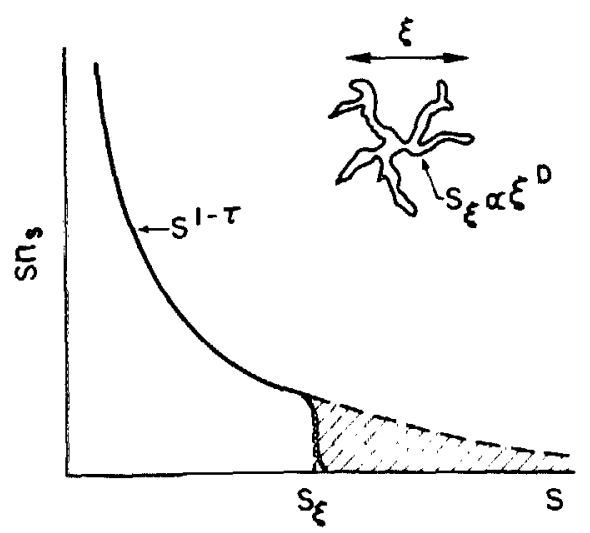

FIG. 1. Assumed distribution $s n_{5}$ of spins in fractal clusters of spin number $s$. Inset shows a schematic fractal cluster of correlation length $\xi$ and volume or spin number $s_{\xi} \propto \xi^{D}$, where $D$ is the fractal dimensionality. 
the shaded area in the figure. That is, the infinite cluster in effect incorporates the largest remaining clusters as it grows. Since this area goes as $(\tau-2)^{-1} s_{\xi}^{2-\tau} \propto \delta|\epsilon|^{\phi / \delta}$, and since the area represents the order parameter which by definition should go as $|\epsilon|^{\beta}$, we recover the standard scaling relation $\beta=\phi / \delta$. Furthermore, we can argue that the volume fraction $\xi^{D} / \xi^{d}$ (where $d$ is the Euclidean dimension) should also go as $|\epsilon|^{\beta}$, which, using $\xi \propto|\epsilon|^{-\nu}$, gives the standard formula for fractal dimensionality $D=d-\beta / v$. With $\phi=\nu D$, this result allows us to calculate $D$ and $v$ from the values for $\phi$ and $\beta$ experimentally determined from equilibrium nonlinear susceptibility measurements. ${ }^{6}$ For example, in the case of GdAl, $\phi=3.3$ and $\beta=0.6$, implying $D=2.5$ and $v=1.3$. We consider these results more reliable than those of Chowdhury and Bhattacharjee ${ }^{5}$ using in part dynamic data and values from different samples.

Finally, we generalize $s_{\xi} \propto|\epsilon|^{-\phi}$ to finite fields by making the standard scaling assumption

$$
\begin{aligned}
& s_{\xi} \propto \xi^{D} \propto|\epsilon|^{-\phi} f_{\xi}\left(H^{1 / y} /|\epsilon|^{\phi}\right), \\
& f_{\xi}(x \rightarrow 0) \rightarrow \text { const, } \quad f_{\xi}(x \rightarrow \infty) \mapsto x^{-1} .
\end{aligned}
$$

The $x^{-1}$ behavior is imposed by the requirement of no critical temperature dependence in the field-dominated limit and implies that $s_{\xi} \propto H^{-1 / y}$, a remarkably simple result. ${ }^{3,15}$ In conjunction with Eq. (2), it implies that both ferromagnetic and spin glass critical clusters in the field-dominated limit have a moment going as $H^{-1}$.

With this background, we are now ready to evaluate the magnetization or susceptibility in various limits for both the ferromagnet and the spin glass. First, in the temperaturedominated limit above $T_{G}\left(\epsilon^{\phi} / H^{1 / y} \rightarrow \infty\right)$, the Langevin function of Eq. (2) reduces to $\mathscr{L}(x) \rightarrow x / 3$. Thus, for a ferromagnet we obtain from Eq. (2) that $\chi \propto \mathrm{s}_{\xi}^{3-\tau}$. Since $s_{\xi} \propto \epsilon^{-\phi}$, $s_{\xi}^{3-\tau} \propto \epsilon^{-\phi+\beta}$, and so, with the standard scaling law $\gamma=\phi-\beta$, we obtain the conventional $\epsilon^{-\gamma}$ divergence of the linear susceptibility. By comparison, for a spin glass in the same limit we obtain, using Eq. (3), $\chi=N \mu_{0}^{2} / 3 k T$, as found by others earlier. ${ }^{1,3}$ This simple result comes from the fact that according to Eq. (2), all spins contribute equally to the linear susceptibility, whether they are aggregated into spin glass clusters or not. By contrast, the general scaling form for the singular susceptibility $\epsilon^{\beta} f\left(H^{2} / \epsilon^{\phi}\right)$ does not by itself preclude the possibility of an $\epsilon^{\beta}$ term in the low-field limit. Therefore, in earlier work, ${ }^{10}$ we have allowed for the possibility of such a term, and indeed we have shown ${ }^{6}$ that any single critical cluster contributes $\xi^{D} / \xi^{d} \propto \epsilon^{\beta}$ to $\chi$. However, our fits to the data always showed the $\epsilon^{\beta}$ term to be within experimental error of zero. ${ }^{10}$ Now we see the reason why: in the net contribution of all the clusters and single spins to the linear susceptibility, only the total number of spins plays a role, and so the critical $\epsilon^{\beta}$ term vanishes.

Next we derive the nonlinear susceptibility of the spinglass in the same limit by expanding the Langevin function to higher order ${ }^{6}$ :

$$
\chi_{n e}=-\left(2 N H^{2} \mu_{0}^{4} / 45 k^{3} T^{3}\right) \kappa_{\xi}^{3-+} \int_{0}^{\infty} d x x^{2-\tau} f_{s}(x) .
$$

As for the linear susceptibility of the ferromagnet, $s_{\xi}^{3-\tau}$ goes as $\epsilon^{-\gamma}$ and thus exhibits the divergence characteristic of the phase transition. The experimentally observed negative sign of the nonlinear susceptibility ${ }^{10} \mathrm{can}$ be seen to arise from the curvature of the Langevin function, as discussed elsewhere. ${ }^{6}$ Now we can also see that because the lower limit of the integral in Eq. (6) gives a zero contribution, it is the largest critical clusters which dominate the nonlinear susceptibility; single spins do not contribute.

Next, we consider the equilibrium susceptibility of a spin glass below $T_{G}$ in zero field. The infinite cluster contributes zero to the volume susceptibility of Eq. (2) because its Langevin function is saturated; so while its moment grows as $\sqrt{s}$, the volume occupied grows as $s$, and $s$ goes to infinity. The smaller clusters, however, give a linear contribution, which, using Eq. (4), is $\left(N \mu_{0}^{2} / 3 k T\right)\left(1-\delta s_{\xi}^{2}-T\right)$. Since $\delta s_{\xi}^{2-\tau} \propto \epsilon^{\beta}$ is just the Edwards-Anderson order parameter $q$, we recover the Fischer formula ${ }^{16} \chi \propto T^{-1}(1-q)$. The physical interpretation of this result is that the susceptibility is reduced by the volume excluded by the infinite cluster. So far we have rederived earlier results.

Finally, we consider the field-dominated limit $\epsilon^{\phi} /$ $H^{1 / y} \rightarrow 0$, where, as discussed in connection with Eq. (5), $s_{\xi} \propto H^{-1 / y}$. Therefore, the argument of the Langevin function in Eq. (2) can be written as $3 a x^{y}$, where $x=s / s_{\xi}$ and $a=\mu_{0} v_{5}^{y} H / 3 k T$ is a number independent of $H$ which we expect to be of order unity. Thus, for a ferromagnet $(y=1)$ we have $M \propto s_{\xi}^{2-\tau}$. Since $s_{\xi}^{2-\tau} \propto H^{1 / \delta}$ in this limit, we recover the usual field-dominated critical behavior. The corresponding spin glass calculation yields

$$
\chi=\mu_{0} N H^{-1} s_{\xi}^{(3 / 2)-\tau} \int_{0}^{\infty} d x x^{(1 / 2)-\tau} f_{s}(x) \mathscr{L}\left(3 a x^{1 / 2}\right) .
$$

Since $\mathscr{L}(x \rightarrow 0) \rightarrow x$, the lower limit of this integral diverges and must be dealt with using Eq. (3). So we add and subtract $x^{1-\tau} f_{s}$ in the integral, multiply and divide by $a$, and obtain

$$
\begin{aligned}
\chi= & \left(N \mu_{0}^{2} / 3 k T\right)\left\{1-s_{\xi}^{2}-\tau \int_{0}^{\infty} d x x^{1-\tau} f_{s}(x)\right. \\
& \left.\times\left[1-\frac{\mathscr{L}\left(3 a x^{1 / 2}\right)}{a x^{1 / 2}}\right]\right\} .
\end{aligned}
$$

Since $\mathscr{L}\left(3 a x^{1 / 2}\right) / a x^{1 / 2}$ is always 1 or less, the brackets are always zero or positive, and thus we obtain a net negative contribution to $\chi$ going as $s_{\xi}^{2-\tau} \propto H^{2 / \delta}$.

To obtain more insight into this result, we approximate $f_{s}$ by a step function, and $\mathscr{L}(x)$ by $x / 3$ up to $x=3$ and by 1 thereafter. In other words, we make a sharp distinction between saturated (field-frozen) and unsaturated clusters. Thus, there are two terms contributing to $\chi$ in Eq. (1):

$\chi=\mu_{0} N H^{-1}\left[\int_{0}^{s_{\xi^{a}}{ }^{2}} d s s^{1-\tau} a s_{\xi}^{-1 / 2}+\int_{s_{\xi}^{a^{-2}}}^{s_{\xi}} d s s^{(1 / 2)-\tau}\right]$,

where the first term represents the unsaturated, the second the saturated, clusters ( $a$ can be assumed to be slightly greater than 1). Transforming the first term using Eq. (3) and the last term using $a$, we obtain

$\chi=N \mu_{0}^{2} / 3 k T\left[1+s_{\xi}^{2-\tau} \int_{a^{-2}}^{1} d x\left(-x^{1-\tau}+a^{-1} x^{(1 / 2)-\tau}\right)\right]$,

where the first two terms now represent the unsaturated 
clusters and the last (positive) term the saturated clusters. The negative term has the form of the number integral for the saturated clusters and therefore it can be interpreted as arising from the volume excluded from the unsaturated clusters. Since $s_{\xi} \propto H^{-2}, s_{\xi}^{2-\tau}$ gives terms going as $H^{2 / \delta}$. Thus, we see a competition between two physical effects contributing to the nonlinear $H^{2 / \delta}$ term in the field-dominated limit: a negative excluded-volume term and a positive saturatedcluster term. Inspection of the integrals in Eq. (10) shows that for all $a$, the negative excluded volume term is larger, though not by much.

This is our principal new result and it has a number of implications. First it indicates that the physical interpretation of the Fischer formula ${ }^{16}$ as a simple excluded-volume effect cannot be extended to finite fields; the saturated cluster contribution must also be taken into account. Secondly, it explains our previously puzzling result ${ }^{6}$ of a positive cluster contribution to $H^{2 / \delta}$ which conflicted with the negative term observed in experiment. Now theory and experiment are in agreement. Thirdly, it has implications for dynamics because the relaxation processes involved in the response of an unsaturated cluster to a change in field or temperature may be different from that of a saturated cluster. If, for example, the negative excluded-volume term responds more rapidly than the positive cluster term, then a decrease in temperature at constant field could cause a negative transient in the susceptibility. Such an effect has indeed been observed recently by Nordblad et al. ${ }^{17}$ The different mechanisms also imply that in the conventional formula for scaling the system's longest time constant $\tau=\tau_{0}\left(\xi / \xi_{0}\right)^{z}, \tau_{0}$, or $z$ will depend on whether the system has saturated clusters or not, that is, they will depend on field and temperature. Nevertheless, as long as $\tau_{0}$ and $z$ obey scaling with the single correlation length $\xi$, one may write the general dynamic scaling law $\tau=\tau_{0}|\epsilon|^{-v z} f\left(H^{2} / \epsilon^{\phi}\right)$. This is just the dynamic scaling law proposed by Bontemps et al. ${ }^{12}$ in their studies of EuSrS. (In their notation, the crossover exponent is $\Delta=\phi / 2$ ). Because static scaling exponents are not available for this spin glass, they did not compare their value of $\phi=2 \Delta=4$ to static exponents, but we comment that this value is not far from values of 3-5 determined in $\mathrm{GdAl}$ and $\mathrm{CuMn},{ }^{6-8}$ consistent with at least a rough universality in the exponent values.

The authors thank particularly $\mathbb{E}$. Gefen for his suggestions concerning the Stauffer distribution and the normalization procedure, but also M. Cyrot, S. Barnes, Y. Imry, A. Aharony, Y. Shapir, and R. Bruinsma for stimulating discussions. One author (A.P.M.) thanks M. Schlenker and the Laboratoire Louis Néel, Grenoble, France, for their kind hospitality during the visit which initiated this work.

${ }^{1}$ D. A. Smith, J. Phys. F 5, 2148 (1975).

${ }^{2}$ K. Binder, Z. Phys. B 26, 339 (1977).

${ }^{3}$ A. A. Abrikosov, J. Low. Temp. Phys. 33, 505 (1978).

${ }^{4}$ A. Mookerjee and D. Chowdhury, J. Phys. F 13, 431 (1983).

${ }^{5} D$. Chowdhury and J. K. Bhattacharjee, Phys. Lett. A 104, 100 (1984).

${ }^{6} B$. Barbara and A. P. Malozemoff, J. Less Common Metals 94, 45 (1983).

${ }^{7}$ A. P. Malozemoff, S. E. Barnes, and B. Barbara, Phys. Rev. Lett. 51, 1704 (1983).

${ }^{8}$ S. E. Barnes, A. P. Malozemoff, and B. Barbara, Phys. Rev. B 30, 2765 (1984).

JJ. Chalupa, S. S. Commun. 22, 315 (1977); 24, 429 (1977).

${ }^{10}$ B. Barbara, A. P. Malozemoff, and Y. Imry, Phys. Rev. Lett. 47, 1852 (1981); A. P. Malozemoff, Y. Imry, and B. Barbara, J. Appl. Phys. 53, 7672 (1982).

${ }^{11} \mathrm{~J}$. Rajchenbach and N. Bontemps, J. Phys. L 44, 791 (1983).

${ }^{12}$ N. Bontemps, J. Rajchenbach, R. Chamberlin, and R. Orbach, Phys. Rev. B 30, 6514 (1984).

${ }^{13}$ T. Mizoguchi, T. R. McGuire, S. Kirkpatrick, and R. J. Gambino, Phys. Rev. Lett. 38, 89 (1977).

${ }^{14}$ D. Stauffer, Phys. Rep. 54, 1 (1979).

${ }^{15}$ C. M. Souloulis and K. Levin, Phys. Rev. Lett. 39, 581 (1977).

${ }^{16}$ K. Fischer, Phys. Rev. Lett. 34, 1438 (1975).

${ }^{17}$ P. Nordblad, L. Lundgren, P. Svedlindh, and O. Beckman (private communication). 